\title{
Acquired Unilateral Nevoid Telangiectasia With Pruritus and Unknown Etiology
}

\author{
Yasmin Amir, MD; Tatyana Nektalova, MD; Orit Markowitz, MD
}

\section{PRACTICE POINTS}

- Unilateral nevoid telangiectasia may present in patients without an underlying hyperestrogenic state.

- Unilateral nevoid telangiectasia may present with symptoms including pruritus.

\section{To the Editor:}

Unilateral nevoid telangiectasia (UNT) is a rare cutaneous disease characterized by superficial telangiectases arranged in a unilateral linear pattern. First described by Alfred Blaschko in 1899, this rare disease has been reported in higher frequency in recent years, with approximately 100 cases published in the literature according to a PubMed search of articles indexed for MEDLINE using the term unilateral nevoid telangiectasia. ${ }^{1}$ Unilateral nevoid telangiectasia can be congenital or acquired; occurs more commonly in women; and typically involves the dermatomal distributions of the trigeminal, cervical, and upper thoracic nerves. Although the pathogenesis of the disease remains unknown, the currently proposed etiology involves hyperestrogenic states, including puberty, pregnancy, and chronic liver disease. ${ }^{2}$ We report a case of progressively worsening, pruritic, unilateral telangiectases of unknown etiology.

A 55-year-old woman presented to our dermatology clinic with progressive red spots involving the right side of the upper body of 3 years' duration. She noted pruritus, and the rash was otherwise asymptomatic. Her medical history was notable for hypertension, dyspepsia, sciatica, uterine fibroids, and a hysterectomy. Her medications included lisinopril, hydrochlorothiazide, tramadol, aspirin, and a multivitamin. The patient did not report the use of oral contraceptive pills or hormone replacement therapy. She also denied the use of cigarettes or illicit drugs but reported occasional alcohol consumption. A review of systems was negative for any constitutional symptoms or symptoms of liver disease. Her family history also was noncontributory.

Physical examination revealed multiple, 1- to 3- $\mathrm{mm}$, telangiectatic macules and patches in a blaschkoid distribution on the right side of the upper chest, back, shoulder, and arm (Figure, $\mathrm{A}-\mathrm{C}$ ). Darier sign was negative. There was no evidence of palmar erythema, hepatosplenomegaly, ascites, thyromegaly, or thyroid nodules. Dermoscopy confirmed the presence of telangiectasia (Figure, D). More specifically, dermoscopy revealed plump telangiectasia with faint pigment in the background, consistent with UNT. Additionally, there was no pink-white, shiny, scarlike background, and vessels were not thin or arborized, further supporting our diagnosis vs other entities included in the differential diagnosis.

Laboratory testing for estrogen levels was within normal postmenopausal limits. A complete blood cell count, basic metabolic panel, hepatic panel, and thyroid stimulating hormone levels all were within reference range. Hepatitis $\mathrm{B}$ and $\mathrm{C}$ virus testing was nonreactive. The diagnosis of UNT was made based on clinical characteristics. The patient then was referred for pulsed dye laser treatment.

Since the first reports of UNT in 1899, it has been described in multiple individually reported cases. The typical description of UNT involves linearly arranged telangiectasia of one side of the body, following either dermatomal or blaschkoid distribution, most commonly

From the Department of Dermatology, Icahn School of Medicine at Mount Sinai, New York, New York.

The authors report no conflict of interest.

Correspondence: Yasmin Amir, MD, Department of Dermatology, Icahn School of Medicine at Mount Sinai, 1 Gustave L. Levy PI, New York, NY 10029 (yasmin.amir@mountsinai.org).

doi:10.12788/cutis.0207 

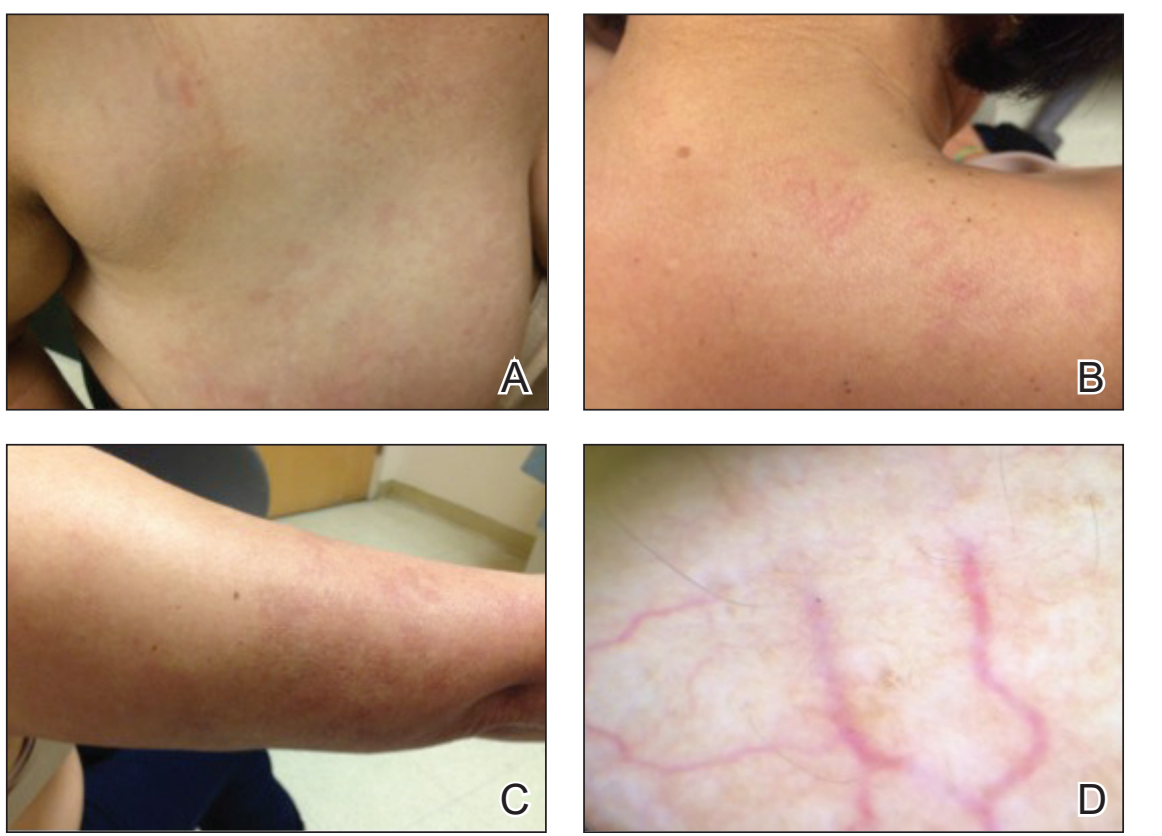

A-C, Multiple, 1- to 3-mm, telangiectatic macules and patches in a blaschkoid distribution on the right side of the upper chest, back, and right arm, respectively. D, Dermoscopy revealed plump telangiectases with faint pigment in the background, consistent with the diagnosis of unilateral nevoid telangiectasia. along the C3 and C4 dermatome. In 1970, Selmanowitz ${ }^{3}$ divided the diagnosis into 2 categories: congenital and acquired. The congenital form is less common overall, seen more frequently in males, and occurs in direct relation to the neonatal period. ${ }^{4}$ The acquired form that is more common overall and seen more frequently in females is suggested to be due to hyperestrogenic states. Most reports of the acquired form involve some underlying pathology that may lead to higher estrogen states. In a review article published in 2011, Wenson et al ${ }^{1}$ summarized the reported cases to date. The authors found that out of close to 100 cases reported, 26 acquired cases were associated with pregnancy and 23 with puberty. They further found 10 cases associated with hepatic disease, 2 associated with hormonal contraceptive pills, 1 associated with hyperthyroidism, and 1 associated with carcinoid syndrome. ${ }^{1}$

Interestingly, a more varied presentation of disease has been reported, as cases are now being reported in healthy patients with no comorbidities or reasons for hyperestrogenism. ${ }^{5}$ In fact, presentations in healthy adult men have led some authors to believe that estrogen may not play a major role in the pathogenesis of the disease..$^{5-8}$ Reports of 16 cases of UNT have indicated no association with hyperestrogenic states. ${ }^{1}$ Because the etiology remains unknown, individual cases both supporting and refuting the hypothesis of estrogen-driven vessel inflammation may drive the investigation of further explanations.

Because UNT usually is asymptomatic, treatment options are largely based on improvement in appearance of the lesions. The pulsed dye laser (PDL) has shown success in treatment of lesions, as Sharma et al, ${ }^{9}$ reported resolution of lesions in 9 cases. These cases were not without side effects, as some patients did experience reversible pigmentary changes. Other studies have validated the use of PDL for cosmetic improvement of UNT; however, some studies have noted the recurrence of lesions after treatment. ${ }^{10}$

Our case provides another unique presentation of UNT. Our patient was a healthy adult woman with no hyperestrogen-based etiology for disease. Importantly, our patient also represented a rare instance of UNT presenting with symptoms such as pruritus, though UNT classically is described as an asymptomatic phenomenon. In our patient, treatment with PDL was suggested and believed to be warranted not only for cosmetic improvement but also in light of the fact that her lesions were symptomatic.

\section{REFERENCES}

1. Wenson SF, Jan F, Sepehr A. Unilateral nevoid telangiectasia syndrome: a case report and review of the literature. Dermatol Online J. 2011;17:2.

2. Wilkin JK. Unilateral nevoid telangiectasia: three new cases and the role of estrogen. Arch Dermatol. 1977;113:486-488.

3. Selmanowitz VJ. Unilateral nevoid telangiectasia. Ann Intern Med. 1970;73:87-90.

4. Karakaş M, Durdu M, Sönmezoğlu S, et al. Unilateral nevoid telangiectasia. J Dermatol. 2004;31:109-112.

5. Jordão JM, Haendchen LC, Berestinas TC, et al. Acquired unilateral nevoid telangiectasia in a healthy men. An Bras Dermatol. 2010;85:912-914.

6. Taşkapan O, Harmanyeri $Y$, Sener O, et al. Acquired unilateral nevoid telangiectasia syndrome. Acta Derm Venereol. 1997;77:62-63.

7. Karabudak O, Dogan B, Taskapan O, et al. Acquired unilateral nevoid telangiectasia syndrome. J Dermatol. 2006;33:825-826.

8. Jucas JJ, Rietschel RL, Lewis CW. Unilateral nevoid telangiectasia. Arch Dermatol. 1979;115:359-360.

9. Sharma VK, Khandpur S. Unilateral nevoid telangiectasia-response to pulsed dye laser. Int J Dermatol. 2006;45:960-964.

10. Cliff S, Harland CC. Recurrence of unilateral naevoid telangiectatic syndrome following treatment with the pulsed dye laser. J Cutan Laser Ther. 1999;1:105-107. 